\title{
Comparative analysis of blood and saliva expression profiles in chronic and refractory periodontitis patients
}

\author{
Bin Zhang ${ }^{1,2}$, Ting Lin² and Hong He $\mathrm{H}^{\text {* }^{*}}$
}

\begin{abstract}
Background: This study aimed to identify characteristic representative genes through a comparative analysis of gene expression profiles in the blood and saliva of chronic periodontitis (CP) and refractory periodontitis (RP) patients to provide new treatment strategies that may be helpful in the treatment of different forms of periodontitis.

Methods: GSE43525 was downloaded from Gene Expression Omnibus. In the dataset, thirteen samples were from blood including 4 controls, 4 CP and 5 RP samples, and ten samples were from saliva including 3 controls, 4 CP and 3 RP samples. After comparing the CP and RP samples, differentially expressed genes (DEGs) between these two types of periodontitis in the blood and saliva samples were identified by an LIMMA package. Then, functional and pathway enrichment analyses were performed by DAVID and KOBAS, respectively. The significantly associated miRNAs in CP and RP were searched by WebGestalt.
\end{abstract}

Results: In total, 213 DEGs in CP and 45 DEGs in RP were identified. Functional enrichment showed that the DEGs of $\mathrm{CP}$ were mainly enriched in ribosome and regulation of apoptosis-related pathways in blood as well as saliva, while the DEGs of RP were significantly enriched in immune responses and response to organic substance-related pathways. Several miRNAs, such as miR-381 and miR-494, were identified as being closely associated with CP. In addition, CD24, EST1, MTSS1, ING3, CCND2 and SYNE2 might be potential targets for diagnosis and treatment of CP.

Conclusion: The identified DEGs and miRNAs might be potential targets for the treatment of chronic and refractory periodontitis.

Keywords: Chronic periodontitis, Refractory periodontitis, Blood, Saliva, Functional and pathway enrichment analysis, MiRNA

\section{Background}

In recent years, periodontal diseases have become a major health problem worldwide. Periodontal diseases are infectious diseases caused by bacteria in the dental plaque [1]. Chronic periodontitis (CP) is an inflammatory destruction of tooth supporting structures that, if

\footnotetext{
* Correspondence: honghehhe@163.com

Highlights

1. In total 213 DEGs in CP and 45 DEGs in RP were identified in blood and saliva tissues.

2. The DEGs were involved in ribosome antigen processing and presentation pathways.

3. CD24 EST1, MTSS1, ING3, CCND2 and SYNE2 might be potential targets for $C P$.

'School and Hospital of Stomatology, Wuhan University, No.237 Luoyu Road, Hongshan District, Wuhan 430079, China

Full list of author information is available at the end of the article
}

left untreated, may lead to tooth loss [2, 3]. CP affects nearly $50 \%$ of the adult population and $60 \%$ of the aged population globally [4]. CP is the most prevalent and destructive disease in adults [5]. In most periodontitis cases, therapeutic success is obtained for long periods of time [6]; however, a small proportion of treated patients, referred to as refractory periodontitis (RP) patients, do not respond well to properly performed conventional therapy and continue to show loss of periodontal attachment $[7,8]$. The existence of specific pathogenic microbiotas may contribute to RP [9]. RP patients are very heterogeneous in terms of their clinical, microbiological and immunological characteristics [10].

$\mathrm{CP}$ is found in the presence of subgingival calculus and local factors [11]. The pathogenesis of $\mathrm{CP}$ is multifactorial 
in nature and results from interactions among bacterial, environmental, immunologic and genetic factors [12]. There is growing evidence that polymorphisms in interleukin 1 (IL1), IL6, IL10, etc. may be associated with CP in certain populations. Several cytokines, including tumournecrosis factor- $\alpha$ (TNF- $\alpha$ ), IL1, IL6, IL8, intercellular adhesion molecule-1 (ICAM-1), monocyte chemoattractant protein-1 (MCP-1), and granulocyte-macrophage colonystimulating factor (GM-CSF), play important roles in the development process of periodontitis. The nuclear factor$\kappa B(N F-\kappa B)$ signalling pathway is involved in parts of the immune response, such as antigen presentation, immune balance and lymphocyte activation, and is significant in periodontitis [13]. CP is initiated and maintained in the first line by complex poly-microbial infection, and the innate susceptibility of the patient is now an accepted critical factor that will determine the destructive character of the disease [14]. An intraradicular infection persisting in the complex apical root canal system and extraradicular infection presenting in the form of periapical actinomycosis can lead to RP [15]. With high incidence and prevalent characteristics, the diagnosis and treatment of $\mathrm{CP}$ and $\mathrm{RP}$ has undoubtedly been a challenge for both clinical investigators and clinicians.

The aim of present study was to compare and analyse these two types of periodontitis at the genetic level in blood and saliva samples and to explore the possible mechanism of periodontitis. Biological microarray analysis was used to analyse the gene expression profile of $\mathrm{CP}$ and RP in different biological fluids with the aim of providing additional treatments and the ability to better distinguish periodontitis.

\section{Methods}

\section{Microarray data}

The research analysis process is shown in Fig. 1 The gene expression profile of GSE43525 [16] was downloaded from Gene Expression Omnibus (GEO), which was based on the platform of the HumanHT-12 V4.0 expression beadchip. Each set of expression profiling data, which is submitted to the GEO database mainly by laboratories all around the world, includes platform information, series information and sample information [17]. A total of 13 samples (including 4 control samples, 4 CP samples and 5 RP samples) were obtained from the blood, and 10 samples (including 3 control samples, 4 $\mathrm{CP}$ samples and 3 RP samples) were from the saliva according to the website information (http://www.ncbi.nlm. nih.gov/geo/query/acc.cgi?acc=GSE43525). These samples were chosen from patients of different sexes and ages. All of the patients were from the Faculty of Dentistry, University of Toronto, Toronto, ON and provided informed consent. In additon, GSE43525 was deposited by Lakschevitz et al. whose study was approved by the Office of Research

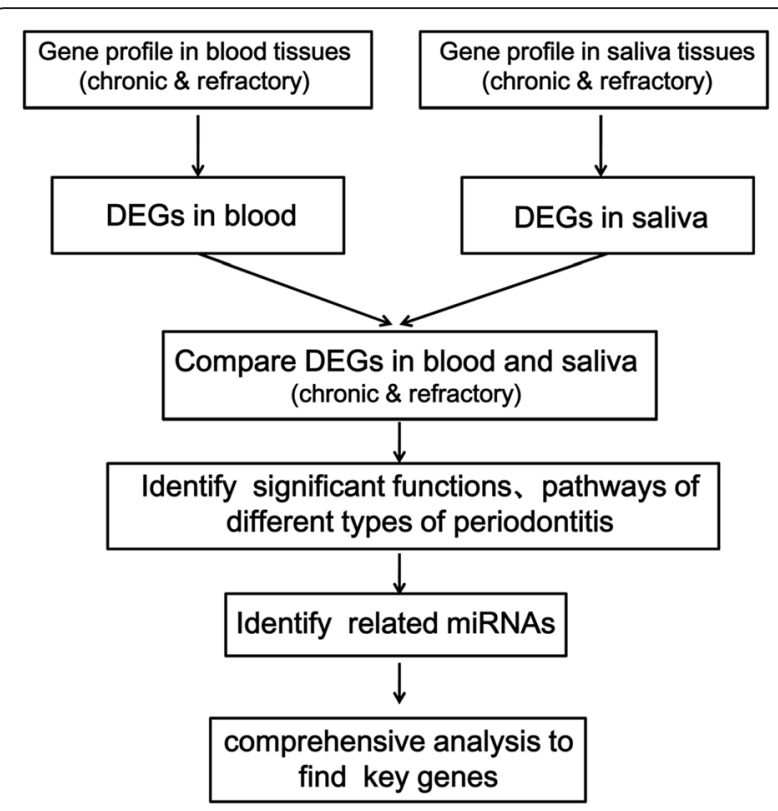

Fig. 1 The research analysis process of blood and saliva tissues

Ethics (ORE) at the University of Toronto (Protocol \# 25698) [16].

\section{Comparative analysis of chronic and refractory periodontitis samples from blood and saliva}

In accordance with the descriptions and characteristics of the original samples, the blood and saliva samples from the CP and RP patients were used for gene expression profile analysis.

\section{Data preprocessing and identification of differentially expressed genes (DEGs)}

The probe-level data were converted into the expression measures. For each sample, the expression values of all probes for a given gene were reduced to a single value by taking the average expression value and then $\log 2-$ transformation was conducted [18]. The linear model of microarray data (LIMMA) package in R [19] was used to identify the DEGs of CP and RP in the blood and saliva compared with normal controls. The $p$-value $<0.05$ and $\mid \log$ fold change (FC) $\mid>1$ were used as the cut-off criteria.

\section{Functional enrichment analysis of DEGs}

Gene enrichment analysis is an analytical strategy using a set of similar or related genes as a whole by calculating the overall significance of changes in gene expression to identify whether the biological function or properties change. This strategy could greatly reduce the dimension of data analysis and makes the analysis process closer to biological problems, therefore this method is widely used in microarray technology [20]. Currently, there are many 
tools that can provide enrichment analysis of gene function. Among them, Database for Annotation, Visualization and Integrated Discovery (DAVID) is the most popular [21]. DAVID provides a comprehensive set of functional annotation tools for investigators to understand the biological meaning behind a large list of genes. We performed a Gene Ontology (GO) enrichment analysis in terms of biological progress (BP). The false discovery rate $(\mathrm{FDR})<0.05$ was used as the cut-off criterion.

\section{Pathway analysis of DEGs}

To better understand and further study the biological functions, the KEGG Orthology Based Annotation System (KOBAS) [22] was used to conduct the pathway annotation and enrichment analysis based on a hypergeometric distribution. The $p$-value $<0.05$ was used as the cut-off criterion.

\section{Searching for significantly associated microRNAs}

MicroRNA (miRNA) broadly participate in many biological processes and can be used as a biomarker for the diagnosis of a variety of diseases [23, 24]. We used the WEB-based Gene Set Analysis Toolkit (WebGestalt) to calculate the association between DEGs and miRNAs in $\mathrm{CP}$ and RP $[25,26]$. The $p$-value $<0.05$ was used as the cut-off criterion.

\section{Results}

\section{Identification of DEGs}

After preprocessing the microarray data, the LIMMA package was used to screen the DEGs. A total of 213 DEGs in CP (including 76 up-regulated and 14 downregulated genes in the blood as well as 94 up-regulated and 29 down-regulated genes in the saliva) and 45 DEGs in RP (including 13 up-regulated and 7 down-regulated genes in the blood, as well as 10 up-regulated and 15 down-regulated genes in the saliva) were identified (Fig. 2).

\section{Functional enrichment analysis of DEGs}

To study the functions of DEGs in CP and RP in blood and saliva, the functional enrichment analysis of all these DEGs were performed by DAVID. The results showed that DEGs in the blood of CP patients were involved in translation-related BP terms (Table 1a). Meanwhile, the DEGs in the blood of RP patients were mainly enriched in immune response processes (Table 1b). Moreover, the DEGs in the saliva of CP patients were mainly involved in the regulation of apoptosis and cell death (Table 1c). In addition, the DEGs in the saliva of RP patients were mainly enriched in genes responsive to organic substances and bacterium (Table 1d).

\section{Pathway analysis of DEGs}

To further understand the involved pathways of each set of DEGs, a pathway enrichment analysis was further performed. A total of 10 pathways were enriched for the DEGs (Table 2). The DEGs of the blood of CP patients were enriched in two pathways, the most significant being ribosome $(p=2.24 \mathrm{E}-10)$, which involved 11 DEGs. The DEGs in the blood of RP patients were mainly involved in five pathways, the most significant of which were cell adhesion molecules (CAMs). Meanwhile, the DEGs in the saliva of CP patients were enriched in the pathway of antigen processing and presentation. The DEGs in the saliva of RP patients were involved in tryptophan metabolism and aldosterone-regulated sodium reabsorption.

\section{MiRNA-target gene analysis}

WebGestalt was used to analyse the related miRNAs in $\mathrm{CP}$ and RP. Table 3 shows the relationship between
A

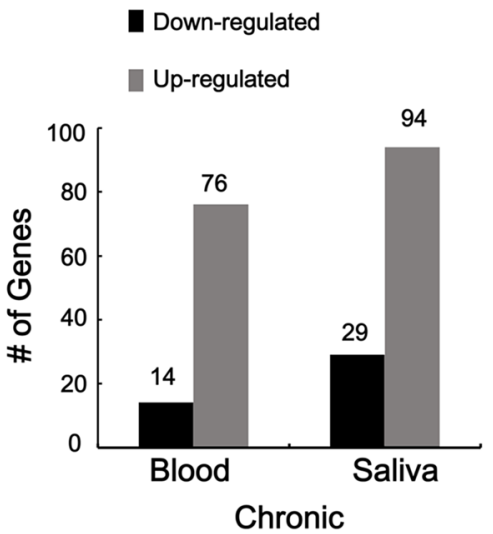

B

Down-regulated

Up-regulated

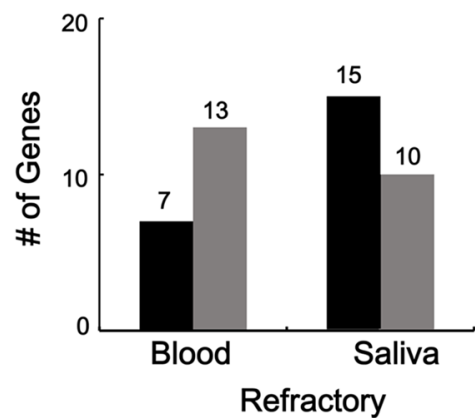

Fig. 2 a: Up-regulated and down-regulated genes in the blood and saliva of patients with chronic periodontitis; b: Up-regulated and down-regulated genes in the blood and saliva of patients with refractory periodontitis. black colour: down-regulated genes; grey colour: up-regulated genes 
Table 1 Gene Ontology enrichment analysis of differentially expressed genes of chronic and refractory in blood and saliva

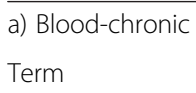

GO:0006414 translational elongation

GO:0006412 translation

GO:0006968 cellular defense response

b) Blood-refractory

Term

GO:0006955 immune response

c) Saliva-chronic

Term

GO:0042981 regulation of apoptosis

GO:0043067 regulation of programmed cell death

GO:0010941 regulation of cell death

GO:0008202 steroid metabolic process

d) Saliva-refractory

Term

GO:0010033 response to organic substance

GO:0009617 response to bacterium

GO:0019439 aromatic compound catabolic process

GO:0008286 insulin receptor signaling pathway

$$
\begin{array}{cl}
\text { Count } & P \text {-value } \\
11 & 8.07 \mathrm{E}-11 \\
13 & 9.52 \mathrm{E}-08 \\
6 & 1.42 \mathrm{E}-05
\end{array}
$$

Count $P$-value

$4 \quad 3.16 \mathrm{E}-02$

Count $P$-value

$12 \quad 2.64 \mathrm{E}-03$

$12 \quad 2.85 \mathrm{E}-03$

$12 \quad 2.93 \mathrm{E}-03$

$6 \quad 3.90 \mathrm{E}-03$

Count P-value

$5 \quad 8.71 \mathrm{E}-03$

$3 \quad 2.13 \mathrm{E}-02$

2 2.34E-02

$2 \quad 4.29 \mathrm{E}-02$

GO:0045787 positive regulation of cell cycle

miRNAs and DEGs. No target genes were found in the RP samples; however, there were 4 and 5 DEGs related to the miRNAs in the blood and saliva of CP patients, respectively. We found that cluster of differentiation 24 (CD24), esterase 1 (EST1), and metastasis suppressor 1 (MTSS1) were target genes for CP in blood, while, inhibitor of growth family member 3 (ING3), cyclin D2 (CCND2) and synaptic nuclear envelope protein 2 (SYNE2) were target genes for $\mathrm{CP}$ in saliva. The most significantly related miRNAs were miR-381 (which targets ETS1 and MTSS1) and miR-494 (which targets ING3, CCND2 and SYNE2).

\section{Discussion}

Periodontitis is a multifactorial infectious inflammatory condition characterized by a destructive inflammatory process affecting the supporting tissues of the tooth and resulting in alveolar bone resumption, periodontal pocket formation and eventually tooth loss [27]. CP is a typical disease that has a relatively mild phenotype and a slow progressing and chronic nature [28]. Functional enrichment showed that DEGs of RP were significantly enriched in immune responses. Additionally, persistent host inflammatory immune responses against pathogens results in the destruction of soft and mineralized periodontal tissues
Table 2 The significanlty enriched pathways by differentially expressed genes

\begin{tabular}{lc}
\hline a) Blood-chronic & \\
Pathway & $P$-value \\
hsa03010:Ribosome & $2.24 \mathrm{E}-10$ \\
hsa05332:Graft-versus-host disease & $3.06 \mathrm{E}-02$ \\
b) Blood-refractory & \\
Pathway & $P$-value \\
hsa04514:Cell adhesion molecules (CAMs) & $6.35 \mathrm{E}-03$ \\
hsa05330:Allograft rejection & $3.49 \mathrm{E}-02$ \\
hsa05332:Graft-versus-host disease & $3.78 \mathrm{E}-02$ \\
hsa04940:Type I diabetes mellitus & $4.06 \mathrm{E}-02$ \\
hsa05320:Autoimmune thyroid disease & $4.92 \mathrm{E}-02$ \\
c) Saliva-chronic & \\
Pathway & $P$-value \\
hsa04612:Antigen processing and presentation & $1.24 \mathrm{E}-02$ \\
d) Saliva-refractory & \\
Pathway & $P$-value \\
hsa00380:Tryptophan metabolism & $4.63 \mathrm{E}-02$ \\
hsa04960:Aldosterone-regulated sodium reabsorption & $4.74 \mathrm{E}-02$ \\
\hline
\end{tabular}

[29]. According to our results, functional level analysis showed that these two types of periodontitis could cause significant expression changes in the immune defencerelated genes in the blood and saliva. As soon as the pathogens invade the periodontal tissue, the immune system uses a variety of methods including humeral immunity and cellular immunity to avoid the occurrence of periodontitis [30]. In our study, we obtained DEGs and their corresponding miRNAs in the blood and saliva of CP patients. Through searching for significantly associated microRNAs for the DEGs, the target genes including

Table 3 Target genes and their corresponding miRNAs in different groups

\begin{tabular}{clll}
\hline a) Blood chronic & & & \\
MiRNA & ID & P-value & Target genes \\
hsa_CTTGTAT,MIR-381 & DB_ID:731 & 4.20 E-02 & ETS1, MTSS1 \\
hsa_TGTGTGA,MIR-377 & DB_ID:845 & $4.07 E-02$ & CD24, ETS1 \\
hsa_CAGCAGG,MIR-370 & DB_ID:682 & $2.73 E-02$ & SLAMF6, MTSS1 \\
hsa_ATACTGT,MIR-144 & DB_ID:702 & $4.47 E-02$ & ETS1, ALDH1A3 \\
b) Saliva chronic & & & \\
MiRNA & ID & P-value & Target genes \\
hsa_ATGTTTC,MIR-494 & DB_ID:782 & $4.80 E-03$ & ING3, CCND2, SYNE2 \\
hsa_ACCATTT,MIR-522 & DB_ID:749 & $4.80 E-03$ & ING3, YWHAZ, CCND2 \\
hsa_AGGTGCA,MIR-500 & DB_ID:827 & 1.64E-02 & CREB1, POFUT1 \\
hsa_AGGGCAG,MIR-18A & DB_ID:668 & 3.35E-02 & YWHAZ, CREB1 \\
hsa_TAATGTG,MIR-323 & DB_ID:724 & 4.38E-02 & CREB1, TGFA \\
\hline
\end{tabular}


CD24, EST1, MTSS1 and ING3, CCND2, and SYNE2 were found. MiR-381 (which targets ETS1 and MTSS1) and miR-494 (which targets ING3, CCND2 and SYNE2) were the most significantly related miRNA in the blood and saliva. These miRNAs might function in $\mathrm{CP}$ by regulating their target genes.

CD24 was found to be highly expressed in periodontitis. The consistent high expression of CD24 has been reported in the epithelial attachment to the tooth and in the migrating epithelium of the periodontitis lesion, and titres of serum antibodies auto-reactive with CD24 peptide correlates with the remission of periodontal disease [31]. Functional analysis revealed that MTSS1, which was initially described as a gene missing in invasive bladder cancer cell lines, was an actin binding protein involved in the regulation of actin cytoskeleton dynamics. MTSS1 is shown to be sonic hedgehog (Shh) signaling dependent and synergize with the effects of Gli transcription factors [32]. ING3 is a subunit of the nucleosome acetyltransferase of histone 4 (NuA4) complex, which activates gene expression. ING3 is ubiquitously expressed in mammalian tissues and governs transcriptional regulation and cell cycle control [33]. Although reports on their roles in the progression of periodontitis are rare, we speculated that these genes might play key roles in periodontitis. EST1, MTSS1, ING3, CCND2 and SYNE2, as well as their corresponding miRNAs, might be potential therapeutic targets for periodontitis. The serum antibody titres were increased in all patients with periodontitis to some degree [34]. The genetic susceptibility factors of periodontitis and quick identification of susceptible periodontitis patients will contribute to the prevention and treatment of periodontal disease.

\section{Conclusions}

In summary, our data provides a comprehensive bioinformatics analysis of DEGs in the blood and saliva of CP and RP patients. A total of 213 DEGs in CP and 45 DEGs in RP were identified. CD24, EST1, MTSS1, ING3, CCND2 and SYNE2 may have the potential to be used as targets for periodontitis diagnosis and treatment.; however, more research is still needed to validate our findings.

\section{Abbreviations \\ BP: biological progress; CAMs: cell adhesion molecules; CP: chronic periodontitis; DEGs: differentially expressed genes; FC: fold change; FDR: false discovery rate; GEO: Gene Expression Omnibus; GO: Gene Ontology; KOBAS: KEGG Orthology Based Annotation System; NuA4: nucleosome acetyltransferase of histone 4; RP: refractory periodontitis; Shh: sonic hedgehog.}

\section{Competing interests}

The authors declare that they have no competing interests and financial interests to disclose.

\section{Authors' contributions}

BZ participated in the design of this study. TL performed the statistical analysis. $\mathrm{HH}$ carried out the study together with $\mathrm{TL}$, and collected important background information. BZ drafted the manuscript. HH conceived of this study, participated in the design and helped to draft the manuscript. All of the authors read and approved the final manuscript.

\section{Acknowledgement}

This work was supported by grants from the National Natural Science Foundation of China (NO. 81170991).

\section{Author details}

${ }^{1}$ School and Hospital of Stomatology, Wuhan University, No.237 Luoyu Road, Hongshan District, Wuhan 430079, China. ${ }^{2}$ Key Laboratory of Oral Medicine, Guangzhou Institute of Oral Disease, Stomatology Hospital of Guangzhou Medical University, Guangzhou 510140, China.

Received: 27 July 2015 Accepted: 10 December 2015 Published online: 24 December 2015

\section{References}

1. TP C. Uses of turmeric in dentistry: An update. Indian J Dent Res. 2009:20:107-9.

2. Shusterman A, Salyma $Y$, Nashef A, Soller M, Wilensky A, Mott R, et al. Genotype is an important determinant factor of host susceptibility to periodontitis in collaborative cross and inbred mouse population. BMC Genet. 2013:14:68.

3. Teumer A, Holtfreter B, Völker U, Petersmann A, Nauck M, Biffar R, et al. Genome-wide association study of chronic pe riodontitis in general German population. J Clin Periodontol. 2013;40:977-85

4. Chapple ILC GR, on behalf of working group 2 of the joint EFP/AAP workshop. Diabetes and periodontal disease:consensus report of the joint EFP/AAP workshop on peri-odontitis and systemic diseases. J Clin Periodontol. 2013;40:S106-12.

5. Luciana M, Shaddox CBW. Treating chronic periodontitis: current status, challenges, and future directions. Clin Cosmet Investig Dent. 2010;2:79-91.

6. Karoussis IK, Salvi GE, Heitz-Mayfield LJ, Brägger U, Hämmerle CH, Lang NP. Long-term implant prognosis in patients with and without a history of chronic periodontitis: a 10-year prospective cohort study of the $|\mathrm{TI}|^{\circledR}$ Dental Implant System. Clin Oral Implants Res. 2003;14:329-39.

7. Colombo APV, Bennet S, Cotton SL, Goodson JM, Kent R, Haffajee AD, et al. Impact of periodontal therapy on the subgingival microbiota of severe periodontitis: comparison between good responders and individuals with refractory periodontitis using the human oral microbe identification microarray. J Periodontol. 2012;83:1279-87.

8. Paster BJ, Boches SK, Galvin JL, Ericson RE, Lau CN, Levanos VA, et al. Bacterial diversity in human subgingival plaque. J Bacteriol. 2001;183:3770-83.

9. Colombo APV, Boches SK, Cotton SL, Goodson JM, Kent R, Haffajee AD, et al. Comparisons of subgingival microbial profiles of refractory periodontitis, severe periodontitis, and periodontal health using the human oral microbe identification microarray. J Periodontol. 2009;80:1421-32.

10. Socransky SSSC, Haffajee AD. Subgingival microbial profiles in refractory periodontal disease. J Clin Periodontol. 2002;29:206-68.

11. Marja L, Laine BGL, Crielaard W. Gene Polymorphisms in Chronic Periodontitis. Int J Dent. 2010;2010:324719.

12. Indriolo SG A, Ravelli P, Fagiuoli S. What can we learn about biofilm host interaction from the study of inflammatory bowel disease. J Clin Periodontol. 2011;38:36-43.

13. Hajime Sasaki NS, Emad AS, Yan X, Ricardo B, Leslie M. 0: 18betaGlycyrrhetinic Acid Inhibits Periodontitis Via Glucocorticoid-Independent NF-KB Inactivation In IL-10 Deficient Mice. J Periodontal Res. 2011:45:757-63.

14. Ph.D. ZFSMSBHH. Study the role of proinflammatory and antiinflammatorycytokines in Iraqi chronic periodontitis patients. J Bagh Coll Dent. 2012;24:164-9.

15. Lingyun Su YG, Chengjun Y, Hanguo W, Qing Y. Surgical endodontic treatment of refractory periapical periodontitis with extraradicular biofilm. Oral Surg Oral Med Oral Pathol Oral Radiol Endod. 2010;110:e40-4. 
16. Lakschevitz FS, Aboodi GM, Glogauer M. Oral Neutrophil Transcriptome Changes Result in a Pro-Survival Phenotype in Periodontal Diseases. Plos One. 2013;8(7):e68983.

17. Edgar R, Domrachev M, Lash AE. Gene Expression Omnibus: NCBI gene expression and hybridization array data repository. Nucleic Acids Res. 2002;30:207-10.

18. Smyth GK, Speed T. Normalization of cDNA microarray data. Methods. 2003;31:265-37.

19. Smyth GK. limma: Linear Models for Microarray Data. 2005.

20. Huang DW, Sherman BT, Lempicki RA. Systematic and integrative analysis of large gene lists using DAVID bioinformatics resources. Nat Protoc. 2009;4:44-57.

21. Huang DW, Sherman BT, Lempicki RA. Bioinformatics enrichment tools: paths toward the comprehensive functional analysis of large gene lists. Nucleic Acids Res. 2009:37:1-13.

22. Xie C, Mao X, Huang J, Ding Y, Wu J, Dong S, et al. KOBAS 2.0: a web server for annotation and identification of enriched pathways and diseases. Nucleic Acids Res. 2011;39:W316-22.

23. Lu J, Getz G, Miska EA, Alvarez-Saavedra E, Lamb J, Peck D, et al. MicroRNA expression profiles classify human cancers. Nature, 2005;435:834-838.

24. Calin GA, Croce CM. MicroRNA signatures in human cancers. Nat Rev Cancer. 2006:6:857-866.

25. Zhang B, Kirov S, Snoddy J. WebGestalt: an integrated system for exploring gene sets in various biological contexts. Nucleic Acids Res. 2005;33:W741-8.

26. Wang J, Duncan D, Shi Z, Zhang B. WEB-based Gene SeT AnaLysis Toolkit (WebGestalt): update 2013. Nucleic Acids Res. 2013:41:77-83.

27. Houshmand B, Rafiei A, Hajilooi M. Influence of cytotoxic T lymphocyte antigen-4 (CTLA-4) gene polymorphisms in periodontitis. Arch Oral Biol. 2012;57:1218-24

28. Mombelli A, Casagni F, Madianos PN. Can presence or absence of periodontal pathogens distinguish between subjects with chronic and aggressive periodontitis? A systematic review. J Clin Periodontol. 2002;29:10-21.

29. Liu Y-CG, Lerner UH, Teng Y-TA. Cytokine responses against periodontal infection: protective and destructive roles. 2010. p. 52-206.

30. French EDS CK, Simon SL, Eklund SM, Chen MC, Klotz LC, Vaccaro K. DNA probe detection of periodontal pathogens. Oral Microbiol Immunol. 2007;:1:58-62.

31. Ping Ye HY, Mary S, Neil H. Ligation of CD24 expressed by oral epithelial cells induces kinase dependent decrease in paracellular permeability mediated by tight junction proteins. Biochem Biophys Res Commun. 2011;412:165-9.

32. Jochen U. The expression of metastasis suppressor MIM/MTSS1 is regulated by DNA methylation. Int I Cancer. 2006;1 19:2287-93.

33. Suzuki S, Nozawa Y, Tsukamoto S, Kaneko T, Imai H, Minami N. ING3 Is Essential for Asymmetric Cell Division during Mouse Oocyte Maturation. Plos One. 2013;8:e74749.

34. Dye BA, Herrera-Abreu M, Lerche-Sehm J, Vlachojannis C, Pikdoken L, Pretz $B$, et al. Serum antibodies to periodontal bacteria as diagnostic markers of periodontitis. J Periodontol. 2009;80:634-47.

\section{Submit your next manuscript to BioMed Central and we will help you at every step:}

- We accept pre-submission inquiries

- Our selector tool helps you to find the most relevant journal

- We provide round the clock customer support

- Convenient online submission

- Thorough peer review

- Inclusion in PubMed and all major indexing services

- Maximum visibility for your research

Submit your manuscript at www.biomedcentral.com/submit 